\title{
Effect of carbon nanotubes on response time of ferroelectric liquid crystals
}

\author{
J. Prakash, ${ }^{1,2}$ A. Choudhary, ${ }^{2}$ D. S. Mehta, ${ }^{1}$ and A. M. Biradar ${ }^{2, *}$ \\ ${ }^{1}$ Instrument Design and Development Center, Indian Institute of Technology Delhi, Hauz, Khas, New Delhi 110 016, India \\ ${ }^{2}$ National Physical Laboratory, Dr. K. S. Krishnan Road, New Delhi 110 012, India
}

(Received 21 October 2008; revised manuscript received 8 April 2009; published 7 July 2009)

\begin{abstract}
We present the results of the fast electro-optic response of multiwalled carbon nanotubes (MWCNTs)-doped deformed helix ferroelectric liquid crystal (DHFLC). The fastening of the response in MWCNTs-doped DHFLC has been attributed to the decrease in rotational viscosity and increase in anchoring energy. The decrease in the former is due to the experience of the torque both by MWCNTs and DHFLC and perturbation of order parameter of the DHFLC while the increase in the latter is due to the $\pi$ - $\pi$ electrons stacking between the MWCNTs, DHFLC molecules, and alignment layers. The increase in conductance in doped cells has also been observed.
\end{abstract}

DOI: 10.1103/PhysRevE.80.012701

PACS number(s): 61.30.-v, 42.70.Df, 42.79.Kr, 77.84.Nh

The field of carbon nanotubes (CNTs) has drawn a great deal of interest in fundamental and applied research since their discovery by Iijima in 1991 [1] because they possess fascinating electrical, dielectric, thermal, mechanical, and electronic properties. The CNTs have proved their importance in employing the devices as field-effect transistors [2], memory storage devices [3], sensors and actuators [4,5], and field emission sources [6]. The doping of CNTs [either single walled (SW) or multiwalled (MW)] in liquid crystals (LCs), in order to have fast electro-optic devices, has been pursued by various groups around the world for improving their electro-optical properties [7-10], dynamic response [11], and other physical parameters $[12,13]$. But much of the reported work has been focused on nematic liquid crystals (NLCs) $[8-10,13]$ and twisted NLC $[7,12]$. The doping of CNTs in ferroelectric liquid crystals (FLCs) is rarely reported in literature. It has also been found that thermotropic [14-16] as well as lyotropic [17] LCs have been used as a solvent providing a simple, versatile, and reproducible way to control the order in SWCNTs and MWCNTs. Some studies have been carried out on the translational motion of CNTs dispersed in NLC under an ac and in-plane field [18,19]. Among many intrinsically physical parameters of LC material, the response time is the most important for addressing the fast LC display devices. Lee et al. [7] demonstrated the effect of CNTs on LC to improve the switching behavior. Faster electro-optical response characteristics of carbonnanotubes-nematic suspension have been studied by Chen et al. [8]. The CNTs-doped LC optically compensated birefringent cells were studied by $\mathrm{Lu}$ and Chien [10] for improvement in response time. The deformed helix FLCs (DHFLCs) are very useful and have much applications in display devices because of their low driving voltage, gray scale generation capability, easily achievable alignment, fast response, etc. $[20,21]$. The bistability or memory effect has also been demonstrated by us $[22,23]$ in DHFLCs. In this brief report, the improvement (almost 50\%) in response time, in MWCNTs (we referred CNTs throughout the paper)-doped DHFLC material has been reported. The improvement in the

\footnotetext{
*Corresponding author; abiradar@mail.nplindia.ernet.in
}

response time has been attributed to the decrease in rotational viscosity and increase in anchoring energy.

The MWCNTs were synthesized by chemical vapor deposition method. The diameter of synthesized MWCNTs ranged 30-50 nm with typical length ranging from $0.3 \mu \mathrm{m}$ to several $\mu \mathrm{m}$. The DHFLC (or FLC)-CNT dispersions were prepared by adding small amount $(0.5 \mathrm{wt} \%)$ of CNTs to material and sonicating the mixture at higher frequencies for $2 \mathrm{~h}$. The effective dispersion of CNTs is a challenging problem in CNTs-based experiments. However, Lagerwall and Scalia [24] suggested some solvent and surfactant for dispersing CNTs efficiently in LC. The sample cells for the present study were prepared using indium tin oxide (ITO) glass plates. The desired (squared) pattern area was 0.45 $\times 0.45 \mathrm{~cm}^{2}$. The thickness of the cell was maintained uniformly around $1.4 \mu \mathrm{m}$ using photolithographic technique. Two types of samples were prepared. In the first type, two electrodes were treated with polymer (Nylon 6/6) and strongly rubbed; we call them strongly treated (ST) cells. In the second type, both electrodes were left untreated (i.e., the polymer was neither coated nor rubbed) and they are called weakly treated (WT) cells. Rubbing in the case of the first type of sample of polymer-coated glass plates was done by using a buffing machine (LCBM, Irwin, U.S.A.) to obtain homogeneous alignment. In this study, a DHFLC (FLC 6304, Rolic, Switzerland) and a FLC material (Felix 17/100) were used. The phase sequence of these materials is as follows:

$$
\begin{aligned}
& -14{ }^{\circ} \mathrm{C} \quad 60.5^{\circ} \mathrm{C} \quad 64{ }^{\circ} \mathrm{C} \\
& \text { cryst } \leftrightarrow \mathrm{Sm} C^{*} \leftrightarrow \mathrm{Sm} A \leftrightarrow \text { iso (FLC 6304), } \\
& -28^{\circ} \mathrm{C} \quad 73^{\circ} \mathrm{C} \quad 77^{\circ} \mathrm{C} \quad 84-87^{\circ} \mathrm{C} \\
& \text { cryst } \leftrightarrow \mathrm{Sm} C^{*} \leftrightarrow \mathrm{Sm} A \leftrightarrow \mathrm{N} \leftrightarrow \text { iso (Felix 17/100). }
\end{aligned}
$$

An impedance analyzer 6540 A (Wayne Kerr, U. K.) was used to measure dielectric permittivity $\left(\varepsilon^{\prime}\right)$ and resistance of the samples in the frequency range $20 \mathrm{~Hz}-1 \mathrm{MHz}$. The tilt angle measurement was carried out by observing the texture under polarizing microscope (Ax-40, Carl Zeiss, Germany) on the application of bias field. The material parameters such as spontaneous polarization, rotational viscosity, and response time were measured by automatic liquid-crystal tester 


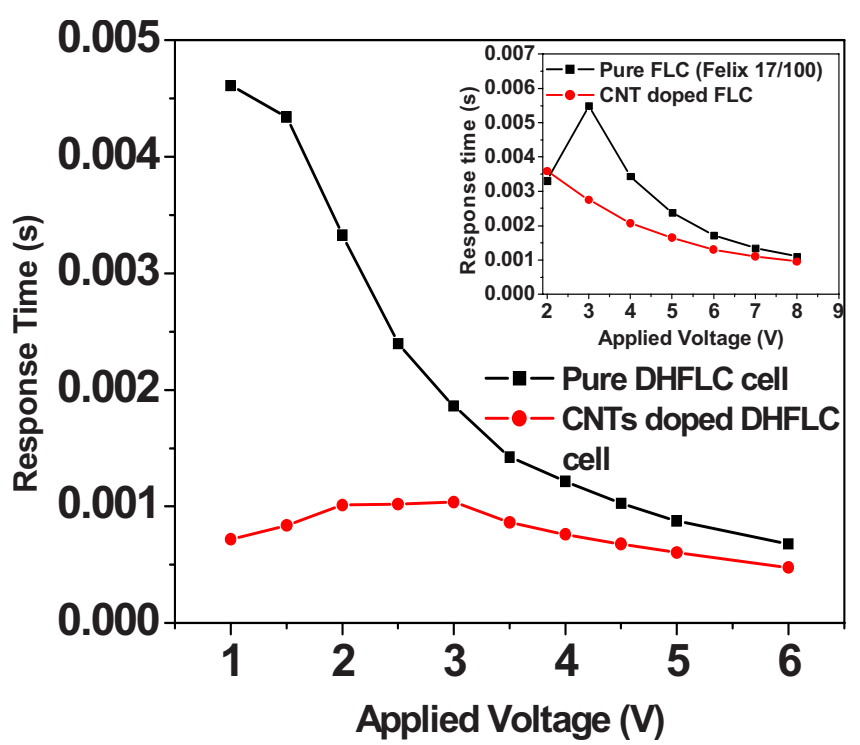

FIG. 1. (Color online) Behavior of response time with applied voltage for ST pure and CNTs-doped DHFLC cells at room temperature. The behavior of the same for FLC is shown in the inset.

(ALCT, Instec, U.S.A.). All the experiments have been carried out at room temperature.

Figure 1 shows the response times of DHFLC and FLC, respectively, as a function of applied voltage. As shown in the figure, after doping CNTs, the response time of DHFLC has been improved (almost 50\% less than that of the pure DHFLC). However, there is no remarkable change in response time of CNTs-doped FLC material as shown in the inset of Fig. 1. The change is more significant particularly at lower voltages as reflected in Fig. 1. This observed improvement in response time in the case of the CNTs-doped DHFLC has been attributed to the decrease in rotational viscosity, increase in anchoring energy, and loosening of DHFLC molecular packing.

First, we take the effect of rotational viscosity because it is directly related with the response time as follows:

$$
\tau=\eta / P_{S} E,
$$

where $\tau$ is response time, $\eta$ is the rotational viscosity, $P_{s}$ is the spontaneous polarization, and $E$ is the applied field, respectively. Figure 2 shows the rotational viscosity as a function of applied voltage. As seen in the figure, the rotational

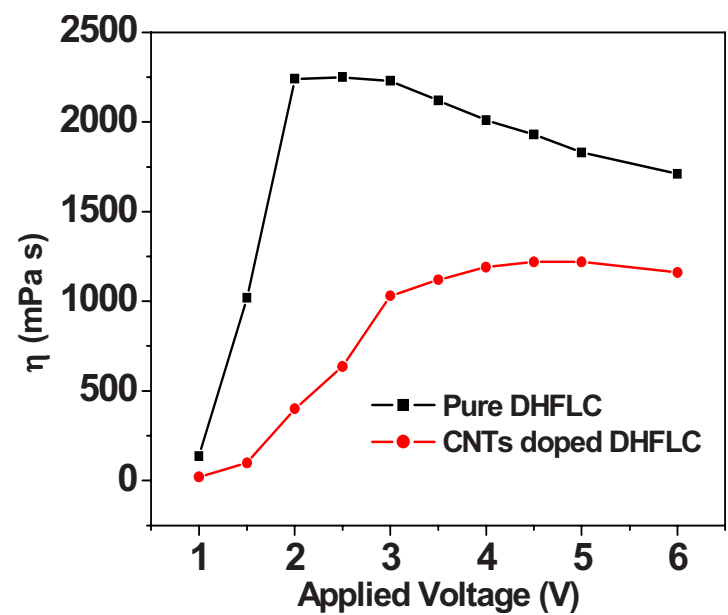

FIG. 2. (Color online) Behavior of the rotational viscosity $(\eta)$ with applied voltage for ST pure and CNTs-doped DHFLC cells.

viscosity decreases after doping CNTs in DHFLC material. This decrease in rotational viscosity is due to two reasons. First, both CNTs and DHFLC molecules experience a torque due to their nontrivial dielectric anisotropies on the application of electric field and hence the decrease in viscosity has been observed. Second, the doping of CNTs in DHFLC perturbs the order parameters (such as tilt angle and spontaneous polarization as these are considered as the order parameters) and, in turn, decreases the rotational viscosity of the material. The change in order parameters has been shown in Fig. 3 [the tilt angle in Fig. 3(a) while as the spontaneous polarization in Fig. 3(b)]. The behavior of tilt angle with applied voltage for pure and CNTs-doped DHFLC material [Fig. 3(a)] clearly shows that there is a substantial difference in tilt angles of pure and CNTs-doped DHFLC cells below the certain voltage i.e., the threshold voltage of around $2 \mathrm{~V}$. The value of the tilt angle in CNTs-doped DHFLC cell is less compared to the pure one below threshold voltage. But after threshold voltage, the tilt angle saturates almost the same in both kinds of cells. The behavior of spontaneous polarization with applied voltage for pure and CNTs-doped DHFLC material [Fig. 3(b)] clearly indicates that the value of the spontaneous polarization in CNTs-doped DHFLC cell is less compared to the pure one. The change in order parameters (tilt angle and spontaneous polarization) confirms the change in rotational viscosity.

Now, we take the effect of anchoring energy on response
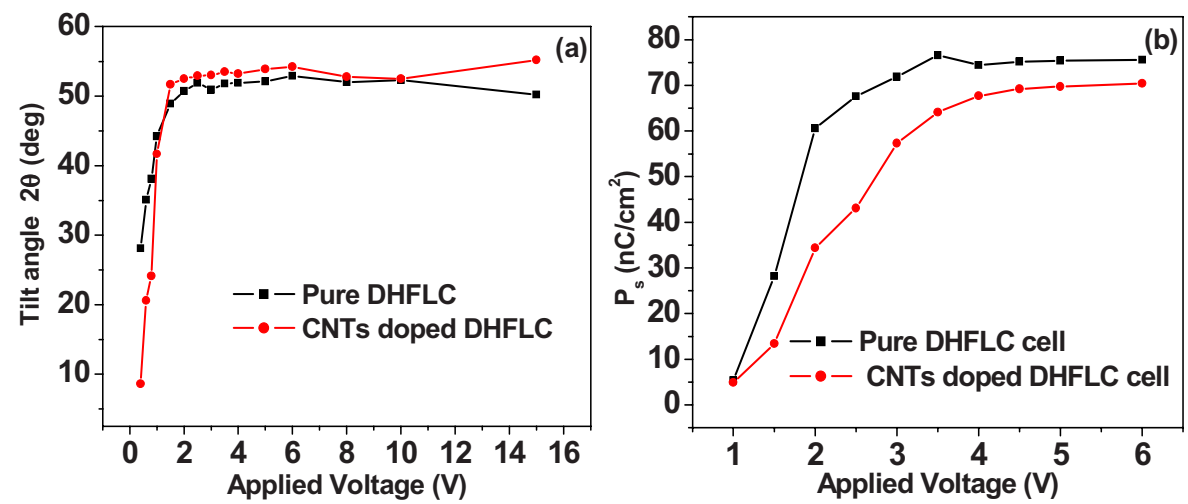

FIG. 3. (Color online) Behavior of (a) the optical tilt $(2 \theta)$ and (b) spontaneous polarization $\left(P_{s}\right)$ with applied voltage for strongly treated pure and CNTs-doped DHFLC cells at room temperature. 


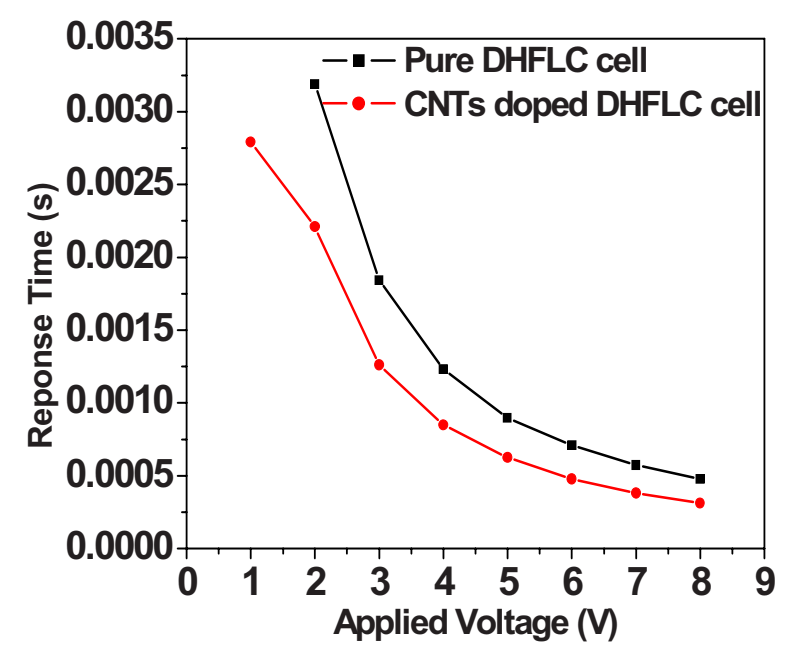

FIG. 4. (Color online) Behavior of response time with applied voltage for WT pure and CNTs-doped DHFLC cells at room temperature.

time in CNTs-doped DHFLC material. The strong anchoring between CNTs and DHFLC molecules increases the elastic energy of DHFLC molecules and therefore is attributed to increase in elastic constant [9] and hence the anchoring energy is altered. It has also been observed that CNTs increase the surface anchoring by 1 order [10]. It has been studied theoretically and validated experimentally that the anchoring energy and cell gap affect the LC response time [25]. The relation of response time with the anchoring energy for strong and weak anchorings, respectively, can be expressed as follows [25]:

$$
\begin{gathered}
\tau \approx \frac{\eta}{K \pi^{2}}\left(d^{2}+\frac{4 d K}{W}\right), \\
\tau \approx \frac{4 \eta d}{W \pi^{2}},
\end{gathered}
$$

where $\tau$ is the response time, $\eta$ is the rotational viscosity, $K$ is bend elastic constant, $d$ is cell gap, and $W$ is the anchoring energy strength coefficient, respectively. Here, the cell gap has been also taken into account. But the cell gap in our case is $\sim 1.4 \mu \mathrm{m}$, so $d$ will not affect much in above equations.

In both the cases (strong anchoring or weak anchoring), the LC response time is inversely proportional to the anchoring energy. It means that if the anchoring energy would have been increased by doping CNTs, the response time should be lowered. We observed the same as reflected from Fig. 4 which shows the response time as a function of applied voltage in WT cells of pure and doped DHFLC materials. As we used WT cells for both, i.e., pure and doped DHFLC materials and observed that, the response time in the case of the doped DHFLC has been shortened. The increase in anchoring energy is due to the $\pi$ - $\pi$ electron stacking between CNTs, surface alignment layer, and DHFLC molecules $[10,11,26]$. It is worth to mention that the change in physical parameters (such as response time, spontaneous polarization, rotational viscosity) is not only due to CNTs but also due to

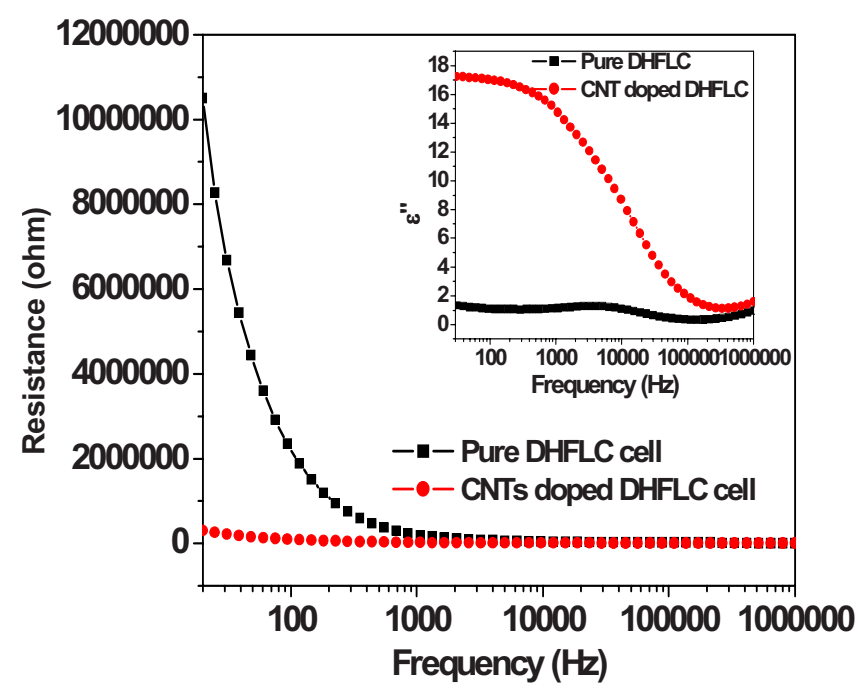

FIG. 5. (Color online) Variation of resistance as a function of frequency in ST pure and CNTs-doped DHFLC cells at room temperature. The variation of the imaginary part of complex dielectric permittivity as a function of frequency has been shown in the inset.

the intrinsic properties of DHFLC material. It is well known that DHFLC materials are short pitched, highly viscous, tightly packed materials. The doping of CNTs looses the packing of DHFLC molecules so they respond fastly as compared to the pure DHFLC material. The loosening of packing of DHFLC is also one of the reasons behind the shortening of the response time.

It has been observed that a minute addition of CNTs in LC medium can increase the conductivity of the medium significantly above the threshold voltage where switching effect was taken into account [11]. Figure 5 shows the resistance of pure and CNTs-doped DHFLC as a function of frequency at a fixed voltage. It is clearly reflected from the figure that the resistance of CNTs-doped DHFLC cell is less compared to the pure DHFLC particularly at lower frequencies. This increase in conductance (or decrease in resistance) is due to increase of imaginary part of dielectric permittivity $\left(\varepsilon^{\prime \prime}\right)$

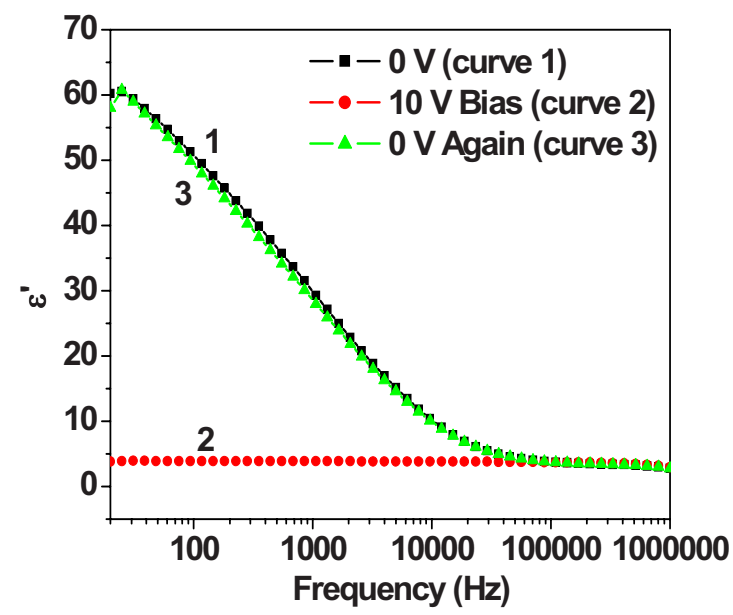

FIG. 6. (Color online) Dielectric permittivity as a function of frequency at room temperature at $0 \mathrm{~V}$ (curve 1$), 10 \mathrm{~V}$ (curve 2), and again $0 \mathrm{~V}$ (curve 3 ) of strongly treated CNTs-doped DHFLC cell. 
about 1 order of magnitude, which happens more significantly in the low-frequency region (the behavior of $\varepsilon^{\prime \prime}$ has been shown in the inset of Fig. 5). As proposed by Dierking et al. [18], the LC director reorientation causes a change in conductance due to anisotropy of LC and observed the increase in conductance with voltage in CNT-doped cells.

As reported earlier, the existence of CNTs in LC does not alter the dielectric permittivity appreciably $[10,12]$. The $\varepsilon^{\prime}$ as a function of frequency in CNTs-doped DHFLC has been shown in Fig. 6. One can clearly see from the figure the change in bias voltage of measuring field from 0 to $10 \mathrm{~V}$; the $\varepsilon^{\prime}$ decreases to the minimum that is due to suppression of Goldstone mode [27]. Again applying $0 \mathrm{~V}$ bias, the $\varepsilon^{\prime}$ appears the same as it was before applying the bias. This confirms that the cell does not remain in stable state and hence no memory effect. The reasons behind observing no memory in CNTs-doped DHFLC are its decrease in rotational viscosity and loosening of molecular packing. It is stabilization of helix deformation that causes memory effect for a prolonged time in pure DHFLC as discussed in our previous report [23]. Here, neither the stabilization of helix deformation nor the compact packing took place in the case of the CNTs- doped DHFLC cell. Therefore, no memory effect was observed in CNTs-doped DHFLC cells.

The fastness of response in CNTs-doped DHFLC material has been observed which has been attributed to the decrease in rotational viscosity and increase in anchoring energy. It has also been observed that the conductance of doped cell increases by several orders of magnitude particularly at lower frequencies. The experimental results presented here not only clarify the fastening of the response but also provide the fundamental concept enabling one to develop electrooptic applications of LC device by incorporation of nanoscale material. It is possible to extend the enabling fastswitching CNTs-doped LC materials to other types of liquidcrystal displays (LCDs) such as blue-phase, cholesteric, supertwisted, polymer-dispersed, surface-stabilized, or electroclinic LCDs.

The authors sincerely thank Dr. Vikram Kumar, Director, NPL, New Delhi, Dr. S. S. Bawa, Dr. I. Coondoo, A. Kumar, and A. Malik for useful discussions. The authors are also thankful to Dr. R. B. Mathur, Carbon Section, NPL for providing CNTs. The authors (J.P. and A.C.) are thankful to CSIR, New Delhi for financial assistance.
[1] S. Iijima, Nature (London) 354, 56 (1991).

[2] R. Martel, T. Schmidt, H. R. Shea, T. Hertel, and Ph. Avouris, Appl. Phys. Lett. 73, 2447 (1998).

[3] T. Rueckes, K. Kim, E. Joselevich, G. Y. Tseng, C. L. Cheung, and C. M. Lieber, Science 289, 94 (2000).

[4] J. Kong, N. R. Franklin, C. Zhou, M. G. Chapline, S. Peng, K. Cho, and H. Dai, Science 287, 622 (2000).

[5] X. Yu, R. Rajmani, K. A. Stelson, and T. Cui, Sens. Actuators, A 132, 626 (2006).

[6] R. H. Baughman, A. A. Zakhidov, and W. A. de Heer, Science 297, 787 (2002).

[7] W. Lee, C. Y. Wang, and Y. C. Shih, Appl. Phys. Lett. 85, 513 (2004).

[8] H. Y. Chen, W. Lee, and N. A. Clark, Appl. Phys. Lett. 90, 033510 (2007).

[9] S. Y. Jeon, S. H. Shin, S. J. Jeong, S. H. Lee, S. H. Jeong, Y. H. Lee, H. C. Choi, and K. J. Kim, Appl. Phys. Lett. 90, 121901 (2007).

[10] S. Y. Lu and L. C. Chien, Opt. Express 16, 12777 (2008).

[11] S. Y. Jeon, K. A. Park, I. S. Baik, S. J. Jeong, S. H. Jeong, K. H. An, S. H. Lee, and Y. H. Lee, NANO 2, 41 (2007).

[12] I. S. Baik, S. Y. Jeon, S. H. Lee, K. A. Park, S. H. Jeong, K. H. An, and Y. H. Lee, Appl. Phys. Lett. 87, 263110 (2005).

[13] H. Y. Chen and W. Lee, Appl. Phys. Lett. 88, 222105 (2006).

[14] M. D. Lynch and D. L. Patrick, Nano Lett. 2, 1197 (2002).

[15] I. Dierking, G. Scalia, and P. Morales, J. Appl. Phys. 97, 044309 (2005).
[16] G. Scalia, J. P. F. Lagerwall, S. Schymura, M. Haluska, F. Giesselmann, and S. Roth, Phys. Status Solidi B 244, 4212 (2007).

[17] G. Scalia, C. von Buhler, C. Hagele, S. Roth, F. Giesselmann, and J. P. F. Lagerwall, Soft Matter 4, 570 (2008).

[18] A. K. Srivastava, S. J. Jeong, M. H. Lee, S. H. Lee, S. H. Jeong, and Y. H. Lee, J. Appl. Phys. 102, 043503 (2007).

[19] I. S. Baik, S. Y. Jeon, S. J. Jeong, S. H. Lee, K. H. An, S. H. Jeong, and Y. H. Lee, J. Appl. Phys. 100, 074306 (2006).

[20] L. A. Beresnev, V. G. Chigrinov, D. I. Dergachev, E. P. Poshidaev, J. Funfschilling, and M. Schadt, Liq. Cryst. 5, 1171 (1989).

[21] J. Funfschilling and M. Schadt, J. Appl. Phys. 66, 3877 (1989).

[22] S. Kaur, A. K. Thakur, R. Chauhan, S. S. Bawa, and A. M. Biradar, J. Appl. Phys. 96, 2547 (2004).

[23] J. Prakash, D. S. Mehta, A. Choudhary, S. Kaur, V. Rathore, and A. M. Biradar, J. Appl. Phys. 103, 044103 (2008).

[24] J. P. F. Lagerwall and G. Scalia, J. Mater. Chem. 18, 2890 (2008).

[25] X. Nie, R. Lu, H. Xianyu, T. X. Wu, and S. T. Wu, J. Appl. Phys. 101, 103110 (2007).

[26] K. A. Park, S. M. Lee, S. H. Lee, and Y. H. Lee, J. Phys. Chem. C 111, 1620 (2007).

[27] S. Hiller, A. M. Biradar, and W. Haase, Phys. Rev. E 53, 641 (1996). 